\title{
Féeries
}

Études sur le conte merveilleux, XVII ${ }^{\mathrm{e}} \mathrm{XIX} \mathrm{X}^{\mathrm{e}}$ siècle

$12 \mid 2015$

À la croisée des genres

\section{Sylvie Catellin, Sérendipité. Du conte au concept}

Paris, Seuil, coll. « Science ouverte », 2014, 265 p.

\section{Emmanuelle Sempère}

\section{(2) OpenEdition}

Journals

Édition électronique

URL : http://journals.openedition.org/feeries/983

DOI : $10.4000 /$ feeries.983

ISSN : 1957-7753

Éditeur

UGA Éditions/Université Grenoble Alpes

\section{Édition imprimée}

Date de publication : 15 octobre 2015

Pagination : 172-177

ISBN : 978-2-84310-306-3

ISSN : 1766-2842

Référence électronique

Emmanuelle Sempère, « Sylvie Catellin, Sérendipité. Du conte au concept », Féeries [En ligne], 12 | 2015, mis en ligne le 15 octobre 2016, consulté le 24 septembre 2020. URL : http://journals.openedition.org/ feeries/983 ; DOI : https://doi.org/10.4000/feeries.983

Ce document a été généré automatiquement le 24 septembre 2020.

(c) Féeries 


\section{Sylvie Catellin, Sérendipité. Du conte au concept}

Paris, Seuil, coll. « Science ouverte », 2014, 265 p.

\section{Emmanuelle Sempère}

\section{RÉFÉRENCE}

Sylvie Catellin, Sérendipité. Du conte au concept, Paris, Seuil, coll. « Science ouverte », 2014, 265 p.

1 Le projet d'interroger la "sérendipité » au croisement de l'histoire des textes et des idées épouse doublement le beau programme annoncé par le titre de cette collection, "Science ouverte »: d'abord par la démarche de son auteure, spécialiste en sciences de l'information et de la communication, éclairant son objet par la philologie, l'analyse littéraire et l'intertextualité ; ensuite par son objet même, la " sérendipité » prônant la disponibilité intellectuelle et une démarche réflexive qui préfère le hasard des rencontres au pas à pas du travail disciplinaire.

De fait, la distance qui semble séparer les «contes» des «concepts» n'est-elle pas purement accidentelle, liée à l'oubli des origines des démarches heuristiques des hommes? Combien de héros de contes, lancés sur la piste du savoir et du bonheur, connaissent un succès à la fois inattendu, étonnant et particulièrement mérité du fait de leur esprit d'initiative, de leur curiosité et de leur intelligence? Parmi eux, les princes de Sérendip, cousins du célèbre Zadig, se caractérisent par le talent particulier de découvrir ce qu'ils ne cherchaient pas, plus exactement, d'exercer leur sagacité sur un phénomène qui les interpelle et qui n'aurait pas dû les intéresser s'ils s'étaient contentés de penser strictement à leurs affaires.

Or, nombre de découvertes dans l'histoire des connaissances humaines se sont faites de cette façon totalement surprenante et inattendue, avec des suites aussi heureuses pour l'humanité qu'en a, pour les princes, le fait de pouvoir décrire un chameau dont ils ont seulement vu (et interprété) les traces ; qu'on les attribue au hasard, à l'intuition ou à la 
curiosité, ces découvertes semblent incompatibles, a priori, avec toute forme de préméditation - autrement dit, elles seraient impossibles dans un contexte où la recherche se voit prescrire des objectifs précis auxquels seront alloués tels moyens et sera concédée telle durée. Ainsi ce livre, s'il annonce une enquête qui va « du conte au concept » de la sérendipité, poursuit-il aussi un objectif plus militant qui fait tout l'objet de la conclusion: pour Sylvie Catellin il est urgent de promouvoir une " politique créative de la recherche » (p. 203) qui non seulement n'entravera pas, mais favorisera la découverte non programmée. Aussi, même si l'on peut regretter, ou sourire d'une certaine complaisance à évoquer le «malaise" des chercheurs soumis aux «financements sur contrat [et à] la complexité des dossiers à remplir qui nécessitent au moins un mois de travail à temps plein » (p. 207), ce livre ouvre-t-il des perspectives tout à fait intéressantes pour la recherche contemporaine, en liant la sérendipité à l'«indisciplinarité » (Laurent Loty), c'est-à-dire à la capacité à s'émanciper des «savoirs transmis » et des «frontières disciplinaires » pour mieux saisir les choses curieuses qui nous entourent, et les interroger.

4 L'auteure de ce livre retrace tout d'abord la transmission du motif fictionnel du chameau perdu et retrouvé, qui est au cœur du conte des Voyages et aventures des trois princes de Sérendip, au monde savant. Elle souligne comme tous les chercheurs qui se sont penchés sur la question ${ }^{1}$ le rôle décisif d'Horace Walpole dans l'histoire du mot et de sa signification; c'est à lui en effet que l'on doit le néologisme serendipity, employé dans une lettre privée pour désigner ces heureuses intuitions qui lui viennent souvent, «à point nommé » (p. 24). Mais ce qui intéresse le plus S. Catellin est de comprendre les conditions de possibilité de ces « découvertes » « accidentelles », et elle fait pour cela le pari d'une archéologie à la fois philologique et épistémologique. Et c'est tout l'intérêt de ce livre, d'étudier l'interaction entre une conception créative et imaginative du savoir et un conte (Walpole lit le conte prétendument traduit du persan du chevalier de Mailly et paru en 1719) - ou plutôt, entre plusieurs contes, car le texte lu par Walpole n'est que l'une des nombreuses versions de ce «motif fictionnel millénaire » dont « il est sans doute vain de chercher à établir l'origine» (p. 36). En effet, les très anciens textes indiens comme les contes arabes médiévaux (en particulier celui de Meydani, l' Histoire des quatre fils de Nizâr, daté de 1284), comme ses transcriptions et variations européennes du $\mathrm{XVI}^{\mathrm{e}}$ au XVIII ${ }^{\mathrm{e}}$ siècles, sous la plume de Christoforo Armeno, de Béroalde de Verville, de Thomas-Simon Gueullette, du chevalier de Mailly et de Voltaire, tous ces textes « retravaillent les rapports entre rationalité et surnaturel, entre interprétation et divination, entre déterminisme et hasard » (p.44). Ce n'est donc pas par souci d'érudition que S. Catellin se penche sur les textes fictionnels qui sont à l'origine du concept, mais parce que "l'étude des variantes [du motif] nous éclaire sur le sens profond du conte de Serendip, et donc aussi sur celui du mot sérendipité » (p. 39). Elle parvient ainsi à rétablir le juste sens de la sérendipité, en relativisant le rôle du « hasard » dans la découverte sérendipienne : celle-ci est «nourrie de savoir» (p. 49, à propos de Zadig) et d'enquête, et relève donc d'une démarche finalement très active, liée à l'exercice d'une liberté et à l'affirmation d'une légitimité. L'archéologie du concept permet d'articuler la sérendipité moderne à une tradition qui chante les vertus d'une "science jointe à l'imagination" (cité p. 45), comme le propose un Lucien de Samosate. L'analyse de S. Catellin est convaincante et intéressante pour nous parce qu'elle s'appuie sur les données internes des textes; en soulignant par exemple le fait que chez Béroalde, Gueullette et Voltaire «le lecteur n'est pas informé, au début de l'histoire, que les trois princes (ou bien le seul Zadig) n'ont pas vu l'animal qu'ils 
décrivent » (p.44), elle montre que la portée épistémologique du motif est liée à la structure narrative : lorsque «le lecteur est amené à mettre lui-même en pratique la sérendipité », le conte « $\mathrm{m}[\mathrm{et}]$ en abyme la quête de connaissance » (p. 46). Des versions plus anciennes du motif, indiennes, talmudiques et arabes, S. Catellin retient surtout la «mise en rapport des liens de causalité et de filiation » (p. 39) : la capacité des princes à remonter d'un fait surprenant à ses causes rend possibles « l'établissement de la vérité et la légitimation d'un droit»-celui que devait leur conférer leur naissance. L'articulation des thématiques de la généalogie et de l'héritage avec la question de la "causalité ", au carrefour de la sémiologie, de la psychanalyse et de la littérature, prend tout son sens dans l'histoire culturelle avec la mise en évidence du rôle qu'a joué la tragédie œdipienne dans l'élaboration du concept de la sérendipité (p. 56-57, analyse prolongée p. 100-106).

Un autre volet de l'enquête philologique concerne Horace Walpole et, là encore, ce sont les textes eux-mêmes qui sont interrogés et pas seulement leur contexte d'écriture. Aussi, après une analyse très précise de la lettre d'Horace Walpole, S. Catellin s'intéresse-t-elle aux autres textes du sagace et excentrique auteur anglais, du Château d'Otrante (1764) aux Contes hiéroglyphiques (1785). Cette étude fine permet de comprendre dans quel sens Walpole a employé le terme de "hasard» (by accidents) dans sa définition de la sérendipité, et de corriger ainsi les dérives sémantiques qu'a connues ensuite la notion. Plutôt que de hasard, il s'agit, chez Walpole, de l'intuition d'une «interférence entre le conscient et le non-conscient dans les processus d'interprétation » (p. 59). Dès lors, le déplacement du concept «de la fiction vers les sciences » (p. 67) entre en relation avec la vogue du récit d'enquête et de la "méthode de Zadig » célébrée à la fois par les scientifiques, comme le biologiste Thomas Huxley, et par les romanciers du detective novel. Le propos de Sylvie Catellin permet de mettre en regard les avancées de la sémiotique dans le dernier tiers du XIX siècle, avec Charles S. Peirce, et le "paradigme policier " illustré par Balzac, Poe et Conan Doyle. Des formes d'inférence logique dégagées par Peirce, l'induction, la déduction et l'abduction (très clairement expliquées p.75), c'est avec cette dernière que la sérendipité entretient une « proximité [...] très forte » (p. 74), l'abduction consistant à «fai[re] une hypothèse sur la règle pour inférer le cas » (p.75): autrement dit, il ne s'agit pas strictement d'une «inférence ", mais plutôt d'un «éclair intuitif» (p. 76) qui devient un « moment préalable » à l'inférence inductive qui, elle, conduira « à la découverte des lois ». Or, si le détective s'attache avec le plus de soin possible à repérer des indices sans connaître ni les causes ni les lois qui les produisent, la solution vient bien souvent d'une "donnée inattendue et surprenante [qui] entre en contradiction avec les certitudes établies » (p. 79, à propos du roman No Name de Wilkie Collins «considéré comme le père du roman policier anglais »). $S$. Catellin identifie là la sérendipité à l'art d'« interpréter les indices" en science comme en littérature, en s'arrêtant tout particulièrement sur le Louis Lambert de Balzac, puis sur La Lettre volée de Poe. La découverte sérendipienne apparaît comme le résultat de l'«attention mobile » (p. 164) de celui qui ne laisse pas sa recherche consciente accaparer toute son attention. L'articulation de l'intelligence d'un héros avec les suggestions nées des phénomènes inattendus et des "coups du sort» explique l'engouement des écrivains, autant que l'intérêt des chercheurs, des médecins et des policiers pour cette qualité rare. Tous s'expriment en termes assez proches et S. Catellin les met habilement en relation. De fait, Balzac affirmant que « le hasard ne visite jamais les sots " (Théorie de la démarche, cité p. 21) ne fait qu'anticiper le discours que prononça Pasteur en 1854, suite à la 
découverte de l'électromagnétisme : «[...] le hasard ne visite que les esprits préparés [...].» (cité p.78) On saura ainsi gré à ce livre de ne pas voir dans les contes les formulations archaïques d'un motif appelé à l'abstraction conceptuelle, mais au contraire de souligner le rôle fondamental de «la littérature [qui] fait réfléchir à la réflexivité par les procédés de mise en abyme, par la démultiplication des personnages, des regards et des voix énonciatives » (p. 116).

Les deux autres chapitres de cet ouvrage, suivant un parcours somme toute chronologique, quittent les écrivains - mais pas complètement la littérature comme on va le voir - pour s'intéresser d'une part aux débats qui ont secoué, dans la première moitié du $\mathrm{xx}^{\mathrm{e}}$ siècle, les milieux savants quant à la part du hasard et de l'inconscient dans la découverte scientifique, et d'autre part à l'histoire de la sérendipité proprement dite depuis le milieu du $\mathrm{xx}^{\mathrm{e}}$ siècle, telle qu'elle a rencontré les domaines de la cybernétique, la notion d'interactivité et les concepts nés du web. L'auteure souligne le rôle majeur, en France, des réflexions de Jean Rostand sur l'importance de l'étonnement dans la découverte scientifique, et de René Taton étudiant les «cas de découvertes par hasard » (p.171), alors même que le mot "sérendipité " peinait à entrer dans la langue et, pire encore, suscitait des définitions paradoxales marquées d'« incomplétude, voire d'incohérence» (p.175). Un tableau des définitions de la sérendipité de Darwin à Didier Houzel, en passant par Edgar Morin (p. 177) accompagne fort utilement l'exposé épistémologique. S. Catellin met en perspective l'intérêt, voire l'enthousiasme des sociétés contemporaines pour la sérendipité avec l'essor sans précédent des « machines à communiquer » (p. 187) - et cette expression ne manquera pas d'évoquer aux lecteurs de Féeries et des travaux d'Yves Citton les «esprits animaux » et les « graines d'hommes » de Tiphaigne de La Roche. Parce qu'elle met en jeu non seulement la curiosité, l'intuition et l'imagination d'un individu, mais aussi les mécanismes d'interaction, le dialogue et la rencontre de plusieurs curiosités, la sérendipité a profondément affaire avec la littérature, dont l'auteure rappelle la définition bakhtinienne (p.188). Pour le prouver, S. Catellin cite le «roman permutationnel de Marc Saporta (1923-2009) intitulé Composition No. $1 »$ (p. 189) dont le fonctionnement nous semble résonner avec celui des contes, à savoir la bifurcation imprévue et la combinaison.

Dès lors, on ne peut s'empêcher de penser qu'il faudrait encore revenir aux contes de la sérendipité étudiés au début de l'ouvrage, avec l'éclairage des études récentes sur les contes auxquelles S. Catellin fait peu appel, pour prolonger les pistes ouvertes par cet ouvrage. L'auteure aurait trouvé des éléments importants pour son étude dans l'appareil critique de l'édition moderne du conte de Mailly, ainsi que dans les travaux sur le conte oriental et en particulier ses modes de composition ${ }^{2}$. On ne saurait cependant reprocher à cet essai son peu de documentation dans le domaine littéraire, puisqu'y figure l'essentiel quant aux sources, et que c'est bien évidemment dans le domaine épistémologique et cognitiviste anglo-saxon que la bibliographie est la plus conséquente. Il faut savoir gré à S. Catellin d'avoir mis en œuvre ce qu'elle plébiscite : par sa démarche, son contenu et ses apports, son ouvrage est un bel exemple d'indisciplinarité ; peut-être doit-on à la sérendipité nombre d'intuitions stimulantes de cet ouvrage, que ce soit dans le domaine littéraire, avec les belles pages sur CEdipe et sur la présence du mythe dans Le Château d'Otrante, ou dans le champ de l'histoire des sciences avec les très intéressantes pages sur Poincaré et le « rôle du travail inconscient dans la recherche mathématique » (p. 161). 
8 Ne devrions-nous pas, à notre tour, nous réinterroger sur la mathématique des contes autrement qu'en termes symboliques et narratifs, et reprendre à nouveaux frais la question des racines mythiques des contes et la circulation des motifs entre la tragédie et le conte merveilleux ? Les livres qui donnent à penser sont toujours de bons livres.

\section{NOTES}

1. Voir le Voyage en Sérendipité dans L. de Mailly, Les Aventures des trois princes de Serendip, suivi de Voyage en sérendipité, par D. Goy-Blanquet, M.-A. Paveau et A. Volpilhac, Vincennes, Éditions Thierry Marchaisse, 2011 ; voir mon compte-rendu, "Au pays de la « sagacité accidentelle » ", Acta fabula, vol. 13, n 5, mai-juin 2012, <http://195.154.199.168/acta/document6980.php>.

2. Jean-François Perrin (dir.), « Le conte oriental », Féeries nº 2, Grenoble, ELLUG, 2005. 